\title{
Growth and characterization of germanium nanowires on a flexible aluminium substrate by electron beam evaporation
}

\author{
R. Rakesh Kumar • K. Narasimha Rao • \\ A. R. Phani
}

Received: 28 February 2011 / Accepted: 7 September 2011/Published online: 23 September 2011

(C) The Author(s) 2011. This article is published with open access at Springerlink.com

\begin{abstract}
For the first time, Germanium (Ge) nanowires have been grown on a gold $(\mathrm{Au})$ coated flexible aluminum (Al) foil substrate in high vacuum $\left(1 \times 10^{-5}\right.$ mbar $)$ by electron-beam evaporation of germanium using the vaporliquid-solid mechanism at a substrate temperature of $380^{\circ} \mathrm{C}$. The grown nanowires have been analyzed for their structural, morphological and chemical properties by employing standard techniques X-ray diffraction, scanning electron microscopy (SEM) and transmission electron microscopy (TEM) and X-ray photoelectron spectroscopy, respectively. X-ray diffraction measurements revealed the formation of cubic Ge phase highly oriented in (111) reflection in plane with the $\mathrm{Al}$ foil substrate. The morphological observations by SEM have shown the randomly grown nanowires with an average length and diameter of $600 \pm 50$ and $100 \pm 10 \mathrm{~nm}$, respectively, for a deposition time of $30 \mathrm{~min}$. TEM investigation revealed single crystalline nanowires with free of defects. The wettability studies by contact angle measurement have confirmed the hydrophobic nature of the Ge NWs film surface with contact angle for water $110^{\circ} \pm 1^{\circ}$. The growth mechanism of $\mathrm{Ge}$ nanowires on $\mathrm{Al}$ foil substrate has also been discussed.
\end{abstract}

R. Rakesh Kumar $(\bowtie) \cdot$ K. Narasimha Rao

Department of Instrumentation and Applied Physics,

Indian Institute of Science, Bangalore 560012, India

e-mail: rakeshr@isu.iisc.ernet.in

\section{A. R. Phani}

Nano-Research for Advanced Materials and Technologies,

Bangalore 560040, India
Keywords Ge nanowires - VLS growth mechanism . Scanning electron microscopy $\cdot$ Flexible substrate . Hydrophobicity · Thin films · e-beam evaporation

\section{Introduction}

Crystalline semiconductor nanowires (NWs) have attracted enormous research interest in recent years, owing to their potential use as building blocks for future nanoscale electronic devices (Cui and Lieber 2001). One dimensional (1D) nanostructures of semiconductors such as $\mathrm{Si}$, $\mathrm{Ge}, \mathrm{GaAs}, \mathrm{GaN}$ and InAs have been grown by different physical vapor deposition (PVD) and chemical vapor deposition (CVD) techniques for possible applications in field-effect transistors (FET) (Liang et al. 2007), light emitting diodes (LED) (Qian et al. 2005), Photo Detectors (Ahn and Park 2007) and Solar cells (Tsakalakos et al. 2007). Among these, 1D semiconductor nanostructures, Germanium NWs are of particular interest for high performance nano electronic devices due to their high carrier mobility and low effective mass (Law et al. 2004; Xiang et al. 2006). Ge NWs have been extensively grown by various methods such as pulsed laser ablation (PLD), chemical vapour deposition (CVD) and vapour transport techniques (Wang 2007). Among the techniques, electron beam evaporation (EBE) technique is quite simple in operation to obtain uniform nanowires compared to CVD technique. It is much inexpensive than molecular beam epitaxy (MBE), and being a non-UHV technique, has much higher throughput which makes it interesting for industrial applications. In the literature, there are only few reports on $\mathrm{Ge}$ nanowires growth by e-beam evaporation technique (Kumar et al. 2010; Pecorea et al. 2010; Sivakov et al. 2007). 
Flexible substrates such as poly (dimethyl siloxane) (PDMS), polymer substrates, poly (ethylene terephthalate) (PET), thin foils of $\mathrm{Al}, \mathrm{Cu}$, have gained increasing attention in the semiconductor industry, due to their light weight and cost in the fabrication process. Ge NWs find applications in fabricating electronic devices (Duan et al. 2003; Lee et al. 2009; Forrest 2004), chemical sensors (Mcalpine et al. 2007). The advantages of Al substrates over Si substrates are flexible, low cost and easy handling. Large area Al substrates are also available commercially. To the best of our knowledge, so far there have been no reports on the growth of Ge NWs on flexible Al substrates by e-beam evaporation. The purpose of depositing on flexible substrate such as $\mathrm{Al}$ foil is, to use the Ge NWs as anode for high density Li ion batteries (Chan et al. 2008; Kim et al. 2010). Experiments are under progress to make device fabrication using Ge NWs grown on $\mathrm{Al}$ foil substrate.

Water repellence (hydrophobic property) is desirable and important for many industrial and biological processes, such as the prevention of the adhesion of snow to antennae (Nakajima et al. 2001), the prevention of contamination (Coulson et al. 2000), stain-resistant textiles and cell mobility (Barady 1994; Blossey 2003). Hydrophobic surfaces are generally prepared by combining the surface roughness at both the micro and nanoscales with low surface-energy materials. This effect occurs in natural systems quite frequently in the case of a lotus leaf, the self-cleaning process is done by water droplets rolling on the surface and removing dirt and debris. Nature accomplishes this phenomenon through the generation of surface topography that displays structures at micro and nanometre scales. Artificial hydrophobic surfaces have been prepared using various strategies (Coffinier et al. 2007; Furstner et al. 2005; Shiu et al. 2004; Oner and McCarthy 2000).

To our knowledge, there are no reports on wettability studies on germanium nanowires film surfaces. Here, we report for the first time, the growth of pure Ge NWs on $\mathrm{Au}$ coated Al foil substrate by e-beam evaporation in high vacuum $\left(1 \times 10^{-5} \mathrm{mbar}\right)$ with hydrophobic property. The morphology, composition, micro structural and wettability properties of Ge NWs have been studied by SEM, XPS, TEM, XRD, Raman and contact angle measurement, respectively.

\section{Experimental procedure}

In the present investigation, to grow Ge NWs by the e-beam evaporation method, pure Ge ingots (purity $99.99 \%$ by Blazers) loaded into water-cooled graphite crucible has been used as a source for e-beam evaporation. The rate and thickness of the deposited Ge has been monitored by a water-cooled quartz crystal monitor. An Al substrate of approximate $160 \mu \mathrm{m}$ thick has been cleaned in an ultrasonic bath with acetone, and de-ionized water and subsequently, loaded into a sputter coating system and deposited with an Au film of $3 \mathrm{~nm}$ thickness.

We have employed BALTEC SCD 500 sputter coating system to deposit gold droplets. For this, after placing the pre-cleaned $\mathrm{Al}$ foil substrate in the chamber, the system has been pumped down to high vacuum $\left(1 \times 10^{-5}\right.$ mbar $)$ and flushed with $\operatorname{Ar}(99.999 \%)$ several times and switch on high voltage for sputtering the gold target. The distance between the substrate and gold target is fixed at $10 \mathrm{~cm}$ to obtain uniform gold film.

The Al substrate was heated to the desired temperature by a in-house built substrate heater and the substrate temperature was calibrated prior to deposition by a thermocouple. Au coated Al substrates have been transferred into the e-beam evaporation chamber and was evacuated using a diffusion pump and rotary pump combination. $\mathrm{Au}$ coated Al substrates have been annealed in situ at $450^{\circ} \mathrm{C}$ under a $1 \times 10^{-5}$ mbar vacuum for 25 min prior to the deposition of $\mathrm{Ge}$ to form $\mathrm{Au}$ droplets. After annealing, the substrate was cooled to $380^{\circ} \mathrm{C}$ and $\mathrm{Ge}$ has been evaporated by the e-beam at the rate of $\sim 0.2 \mathrm{~nm} / \mathrm{s}$ for $30 \mathrm{~min}$. The applied (emission) current to evaporate germanium is $25 \mathrm{~mA}$ and the distance between the source and substrate is $20 \mathrm{~cm}$.

The obtained Ge nanowires on the Al foil substrate has been subjected to adhesion test by using tape. No peeling of the film containing nanowires is observed indicating the good adhesion of the Ge NWs on the Al foil substrate.

The morphological investigation on the Ge NWs has been examined by a scanning electron microscope (Model: SEM FEI Quanta 200). The micro structural properties of the Ge NWs have been evaluated by a transmission electron microscope (Model: TEM, Technai F-30). The structural properties of the films were characterized by X-ray diffraction (XRD, Bruker D8 Advance) and compositional properties were carried out by X-ray Photoelectron spectroscopy (Model: Multilab 2000 Thermo Scientific system) spectroscopy equipped with an Al X-ray source ( $\mathrm{h} v=1,253.6 \mathrm{eV})$. A Phonon confinement study was carried out by Raman spectroscopy (Model: Lab RAM HR Raman system) equipped with a charge coupled device detector $(\mathrm{CCD})$ at working temperature of $-70^{\circ} \mathrm{C}$. The sample is illuminated by the $514 \mathrm{~nm}$ line of an argon ion laser focused at $100 \times$ objective. All the data have been recorded using $2 \mathrm{~mW}$ of laser power and for $20 \mathrm{~s}$ of acquisition. The hydrophobic nature of Ge NWs film has been investigated at ambient atmosphere, by contact angle measurement using deionized water. 


\section{Results and discussion}

\section{SEM}

Tilted view of high and low magnification SEM images of the Ge NWs grown on the Au coated $\mathrm{Al}$ foil substrate has been shown in Fig. 1a, b, respectively. The average length and diameter of the grown NWs were found to be approximately $600 \pm 50$ and $100 \pm 10 \mathrm{~nm}$, respectively. The obtained Ge NWs have grown randomly, which inhibited us to measure the accurate length of the NWs. From SEM images the real density of the NWs was found to be 3-4 wires $/ \mu \mathrm{m}^{2}$. From Fig. 1a, it is clear that each NW has a gold catalytic particle at its ends and confirming the growth of the NWs following vapor-liquid-solid (VLS) mechanism (Wagner and Ellis 1964). The tip containing the Au droplet is indicated by white arrow mark in Fig. 1a, b. The flexible nature of the $\mathrm{Al}$ foil substrate, which contains Ge NWs, can be seen in Fig. 1c. However, we have performed standard tape adhesion test which confirmed good adhesion of the Ge NWs on the surface as well as this is further supported by the Fig. 1c where we have performed measurement on bent sample containing the substrate where we have not observed any peeling of the film. In case of gold film after tape test, we could see gold droplets uniformly throughout surface of the $\mathrm{Al}$ foil. The high magnification SEM image of the gold droplets formed on the $\mathrm{Al}$ substrate, after annealing the Au film of $3 \mathrm{~nm}$ thickness at $450^{\circ} \mathrm{C}$ for $25 \mathrm{~min}$ has been shown in Fig. 1d. It is noteworthy to mention here that no nanowire growth has been observed on bare $\mathrm{Al}$ substrate, eliminating the possibility of catalyst-independent nanowire growth (for bravity SEM images are not shown here).

\section{XRD}

The XRD pattern of the Ge NWs grown on an $\mathrm{Al}$ substrate has been shown in Fig. 2. The measured reflections have been indexed as (111), (220) and (311) planes of the cubic structure of the Germanium (JCPDS card no. 040545) with a lattice parameter $a=b=c=5.65 \AA$ A. Apart from the Ge reflections it has been observed for the used substrate $\mathrm{Al}$ foil. The diffraction planes (111), (200) and (220) for the aluminium foil substrate with cubic structure (JCPDS no. 851327) have been marked with asterisk. No peaks corresponding to any other impurities or catalytic particles
Fig. 1 Ge NWs grown on $\mathrm{Al}$ foil substrate by e-beam evaporation technique. a High and $\mathbf{b}$ low magnification SEM images of Ge nanowires (tilted view) (Au tipped on the surface is marked with white arrows), c the flexible nature of the $\mathrm{Al}$ substrate which contains $\mathrm{Ge}$ NWs (bent view) d High magnification SEM image of gold droplets formed on the $\mathrm{Al}$ substrate after annealing of gold film of $3 \mathrm{~nm}$ thick at $450^{\circ} \mathrm{C}$ for $25 \mathrm{~min}$
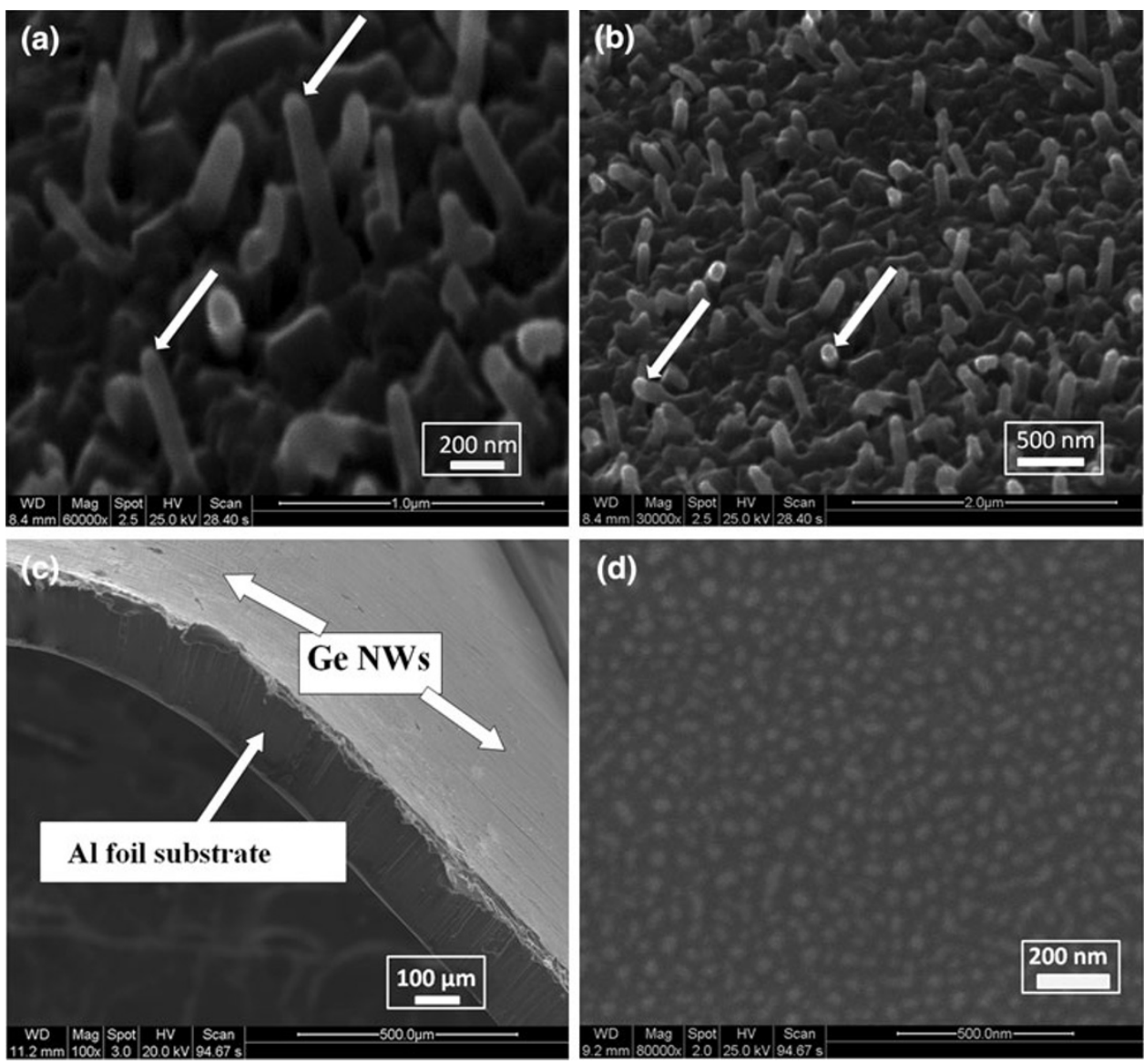


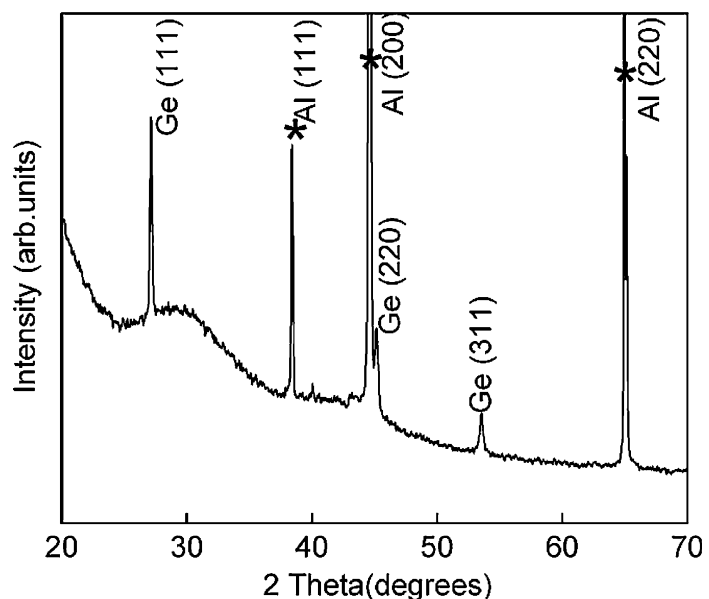

Fig. 2 XRD pattern of the as grown Ge nanowires on flexible $\mathrm{Al}$ foil substrate

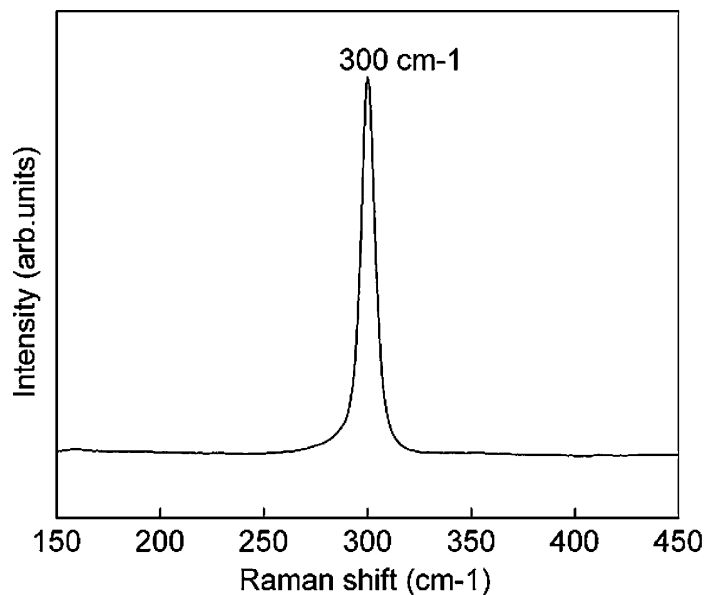

Fig. 3 Raman spectrum of the as grown Ge NWs by e-beam evaporation

were detected. The XRD result confirms that the grown $\mathrm{Ge}$ NWs have shown good crystallinity in nature with sharp and high intensity peaks.

\section{Raman}

Raman spectroscopy is an important tool for investigating the phonon confinement of nanomaterials, as well as to determine the crystalline nature of materials. The Raman spectrum of the Ge NWs grown on Al foil substrate has been shown in Fig. 3. The sharp peak at $300 \mathrm{~cm}^{-1}$ attributed to first order optical phonon contribution of the $\mathrm{Ge}-\mathrm{Ge}$ stretching mode (Pei et al. 2010; Jalilian et al. 2006), and also confirmed the crystalline nature of the Ge NWs, which further supports the XRD measurement. The peak at $300 \mathrm{~cm}^{-1}$ of the NWs is red shift when compared to the bulk $\mathrm{Ge}$ (at $302 \mathrm{~cm}^{-1}$ ) (Jin et al. 1999). The minimum size of the nanowire was observed to be $\sim 100 \mathrm{~nm}$. With this size, no quantum confinement can be observed. The phonon confinement effect can cause an insignificant shift of the peak to the lower energy. Therefore, the red shift of the $\mathrm{Ge}-\mathrm{Ge}$ Raman stretching peak can be understood in terms of the phonon confinement effect (Pei et al. 2010). However, more investigation in this direction is under progress.

\section{XPS}

X-ray photoelectron spectroscopy measurements were performed on the Ge NWs, grown on an Au coated $\mathrm{Al}$ foil substrate. The XPS analysis of the Ge nanowires has been surveyed in the binding energy (BE) range of $0-1,000 \mathrm{eV}$ has been shown in the Fig. 4a. No contamination species were observed within the sensitivity of the instrument apart from the adsorbed atmospheric carbon and Auger peaks of Ge LMM and O KLL. The binding energy calibration of the spectra has been referred to a carbon $1 \mathrm{~s}$ peak located at $284.54 \mathrm{eV}$.

Figure $4 \mathrm{~b}$ shows the XPS spectra of Ge $3 \mathrm{~d}$ core level, obtained from the GeNWs. The core level Ge $3 d$ spectrum indicates the binding energy of $\mathrm{Ge}^{\circ}$ at $29.05 \mathrm{eV}$ and that of $\mathrm{Ge}^{+4}$ at $32.25 \mathrm{eV}$, respectively. This observation is in good agreement with the XPS database (Castain 1992). The presence of a $\mathrm{Ge}^{\mathrm{o}}$ peak implies that the main body of the
Fig. 4 XPS patterns of the asgrown Ge NWs. a General survey spectrum b High resolution XPS core spectrum of Ge 3d level
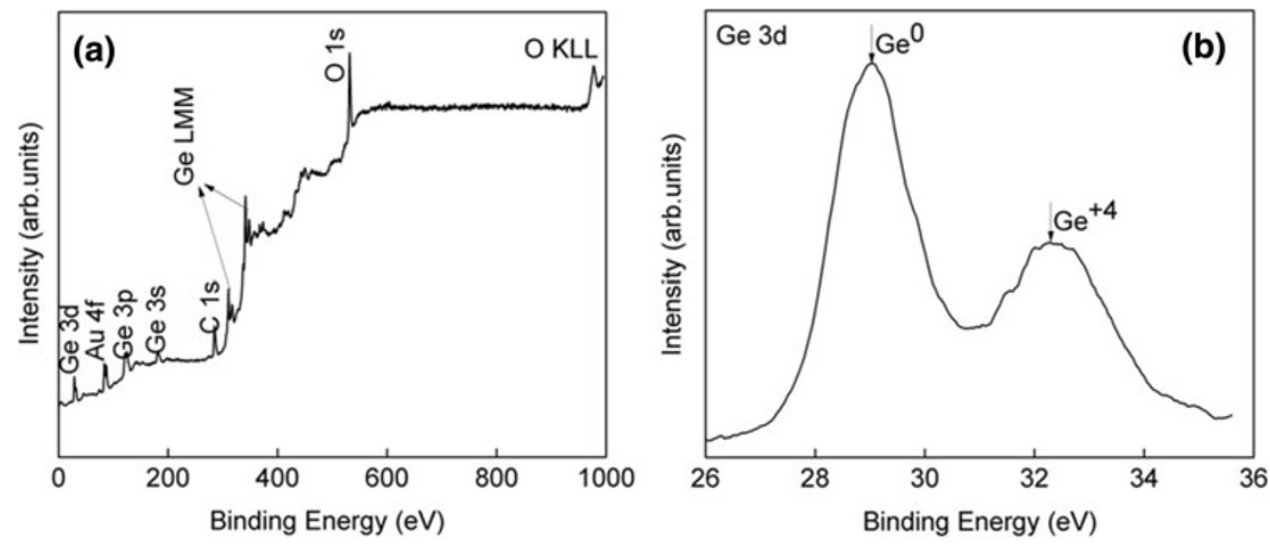
Ge NWs is composed of pure Ge. It is evident that, there is a slight formation of the $\mathrm{GeO}_{2}(32.25 \mathrm{eV})$ on the surface of the formed Ge NWs, and this may be due to exposure of samples to atmosphere (Molle et al. 2006).

\section{Wettability}

Wettability is a very important property of solid surfaces and is governed by the chemical composition, roughness, the manner of the surface preparation and the surface cleanness. The wettability property was characterised by the contact angle water to the solid surface. The formation of a contact angle at the solid-liquid-gas boundary on a rough surface can be described by two models namely Wenzel's equation (Wenzel 1936) and Cassie and Baxter's law (Cassie and Baxter 1944). A hydrophilic surface has a contact angle less than $90^{\circ}$. If the value of the contact angle is greater than $90^{\circ}$, then the surface is hydrophobic and if the value of the contact angle is more than the $150^{\circ}$, then the surface is super hydrophobic. However, to the best of our knowledge there is no report on the wettability studies on Ge NW films. Figure 5 shows the photograph of the water droplet on the Ge NWs film grown on Au coated Al foil substrate. The measured contact angle was $110^{\circ} \pm 1^{\circ}$ for Ge NWs film surface. This confirms that the NWs film surface is hydrophobic in nature. Hydrophobic nature of the surface was caused by air trapped in the rough surface, this being obtained by combining micron and nanometerscale structures induced by nanowires (Coffinier et al. 2007; Cassie and Baxter 1944). Even though Ge itself is hydrophobic, one expects same for Ge NWs films but we have given experimental evidence to the scientific community that Ge NWs film surface showing hydrophobic nature. However, there is no report as on today on hydrophobicity $\left(110^{\circ} \pm 1^{\circ}\right)$ of Ge NWs grown by e-beam technique or any other PVD or CVD technique. This is only a preliminary experiment as our aim to grow vertical Ge NWs with improved wettability towards super-

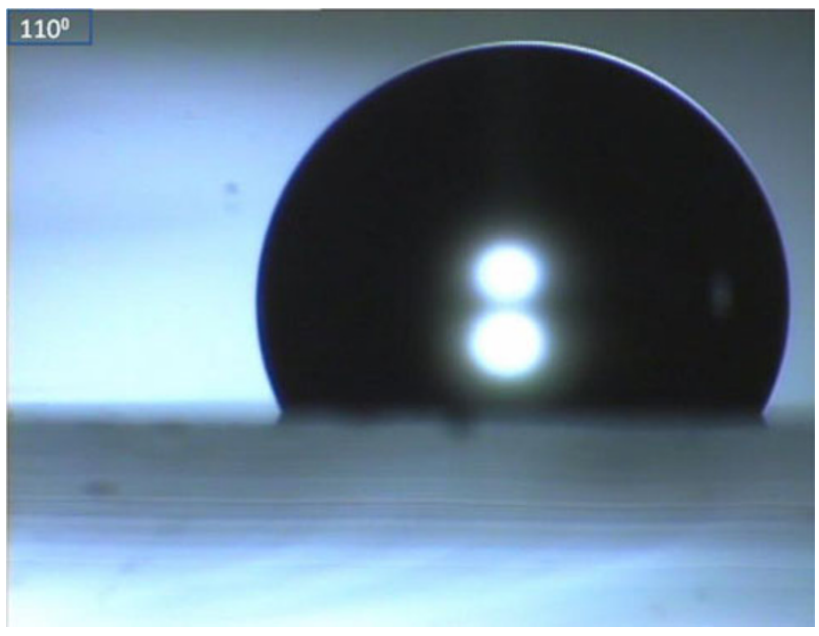

Fig. 5 Photograph of water droplet on Ge NWs surface

hydrophobicity. In fact, there are some references where investigators have modified the surface of $\mathrm{Si}$ NWs by coating some additives (Yung and Gau 2010). Experiments are under progress to increase the contact angle with different growth conditions of Ge NWs.

\section{TEM-EDX}

The Ge NWs were scraped from the $\mathrm{Al}$ foil substrate and moved on to a $\mathrm{Cu}$ grid for TEM characterization. Energy dispersive $\mathrm{X}$-ray analysis has been performed on the tip of the Ge nanowire as well as on the lateral part of the wire which has been depicted in Fig. 6a and Fig. 6b, respectively. Figure $6 a$ it is revealed that the tip of the nanowire contains gold only (Kumar et al. 2010; Sutter and Sutter 2008), whereas the base of the NW contain Ge only. Further, no other impurities or other catalytic particles were observed on the grown NW during TEM analysis. The inset (top left) of Fig. 7 shows the high magnification TEM image of the single Ge NW. This clearly shows the Ge NW with an Au nanoparticle at its end (indicated by white
Fig. 6 Energy Dispersive X-Ray spectrum of a Tip of the nanowire, $\mathbf{b}$ rest of the nanowire
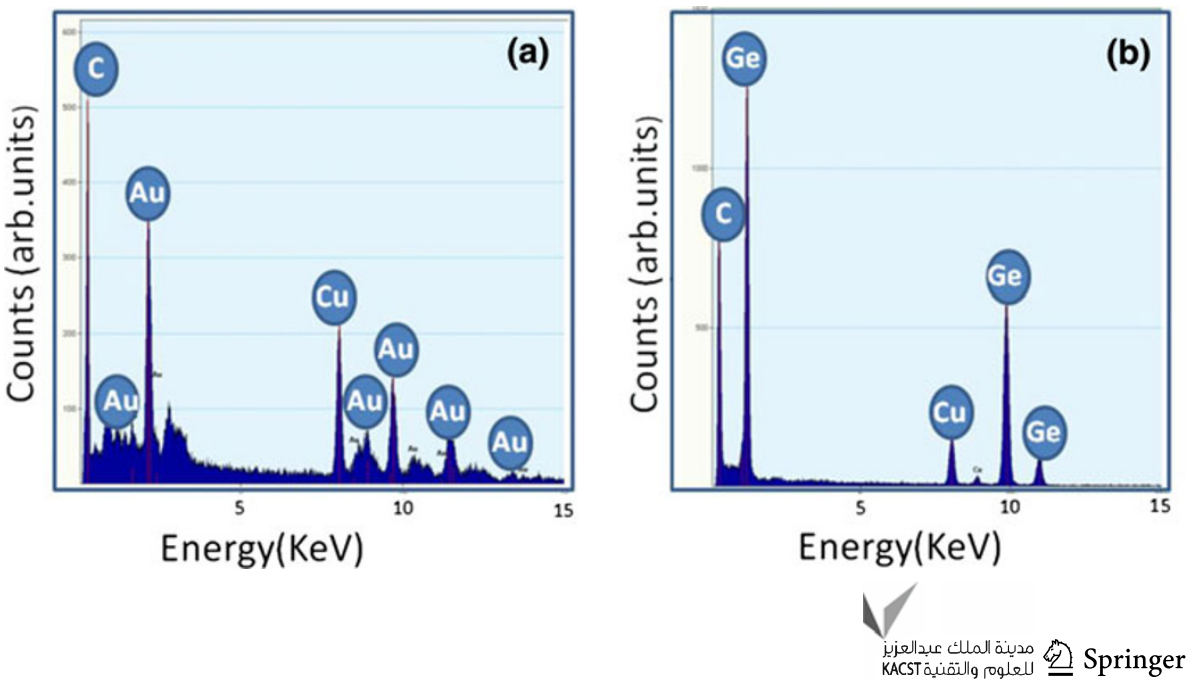


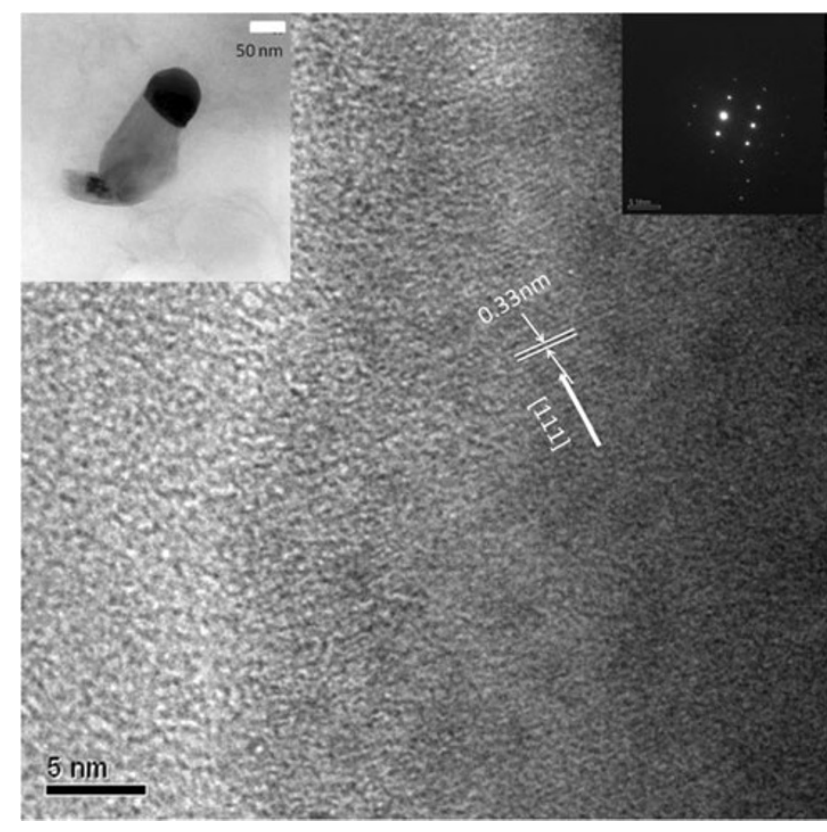

Fig. 7 High Resolution Transmission Electron Microscopy (HRTEM) image of a single germanium nanowire inset top left TEM image of single nanowire with an Au nanoparticle at its end inset top right selected area electron diffraction (SAED) pattern for Ge NW

arrow). The substrate temperature maintained well above the eutectic temperature during growth of NWs, this further confirmed VLS growth of the Ge NWs. The HRTEM image shows the single crystalline nature of the Ge NWs and the nanowire is free of defects. The inset (top right) of Fig. 7 shows the selected area electron diffraction pattern recorded on the NW, which is also supporting the single crystalline nature of the NW. Nanowires have been grown on $\mathrm{Au}$ coated $\mathrm{Al}$ foil substrate having different orientations with respect to substrate, so that there may be different growth directions of nanowires. The lattice spacing in HRTEM in Fig. 7 is found to be $0.33 \mathrm{~nm}$, which corresponds to the "d" spacing of the (111) plane of Ge. The obtained results are in good agreement with observed XRD measurements.

\section{Growth mechanism}

Germanium NWs were grown both perpendicular and inclined with respect to the substrate as shown in the SEM images (Fig. 1a, b). The growth mechanism of Ge NWs grown by e-beam evaporation is similar to the mechanism proposed for $\mathrm{Ge}, \mathrm{Si} \mathrm{NWs}$ grown by e-beam evaporation (Pecorea et al. 2010; Irrera et al. 2009). The growth of NWs can be explained in terms of two competing processes: germanium atoms directly impinging on the gold droplet and germanium atoms adsorbing on the substrate. Germanium atoms impinging on the gold droplet form an alloy with the gold droplet, and causing super saturation of alloy results in a growth of germanium nanowire occurred just at the liquid/solid interface of the alloy and the substrate. This process results in the growth of NWs with a gold particle at the end of the each wire. This mechanism clearly shows the VLS mechanism. Atoms impinging on the substrate also have a fundamental role in the axial growth; in fact, growth conditions allow them to easily diffuse over the sample in such a way that some of the atoms can diffuse along the growing Ge sidewalls. These atoms can be adsorbed at the solid germanium/liquid eutectic interface under the gold droplet. Therefore, they may be having an important contribution to the NWs growth. To our knowledge, as on today in the literature, the maximum length grown with molecular beam epitaxy, electron beam evaporation methods is about 2 micrometer (Pecorea et al. 2010). The main reason for not getting lengthy nanowires is adatom diffusion length of growth species. It plays a key role in nanowires growth by EBE (Artoni et al. 2011; Xu et al. 2011). Initial stage of nanowire growth depends on growth species which deposit on the substrate contribute to nanowire growth. When length becomes larger than the diffusion length, then the length of the nanowire depends mainly on the diffusion of adatoms fall that adsorb directly onto the NW side walls or on the catalyst particle surface. Therefore, after few nm nanowire growth, growth become slow and at the same time 2D layer deposit increases on the substrate, and nanowire grows by collecting growth species from the sidewalls and catalyst. If we increase time of deposition gold diffuse away from the original seed particle, as a result, the nanowire cannot grow in lengthy any more, but over growth on the side walls occurs.

\section{Conclusion}

For the first time, Ge nanowires have been successfully grown on a flexible $\mathrm{Al}$ foil substrate by e-beam evaporation technique under high vacuum $\left(1 \times 10^{-5} \mathrm{mbar}\right)$ at $380^{\circ} \mathrm{C}$ substrate temperature using $\mathrm{Au}$ as a catalyst. SEM measurements indicated that the grown Ge NWs have been oriented in a random direction with respect to the substrate. XRD and Raman studies revealed the crystalline nature of the NWs and also the phonon confinement effect in $\mathrm{Ge}$ NWs. XPS studies revealed that the grown NWs are bit oxidised due to exposure of the samples to the atmosphere. From the TEM studies, the growth of the NWs follows the VLS mechanism. The as grown nanowires were single crystalline in nature and free of defects. Contact angle measurement revealed the hydrophobic nature of the $\mathrm{Ge}$ NWs film surface. Experiments are under progress to grow the Ge NWs in vertical direction perpendicular to the 
substrate with minimum length of 1 micron for practical applications in high speed electronics, high density Li ion batteries, low $\mathrm{k}$ dielectric materials.

Acknowledgments Authors would like to acknowledge Nano Center-Indian Institute of Science, Bangalore for providing the HRSEM and TEM-EDX facility.

Open Access This article is distributed under the terms of the Creative Commons Attribution License which permits any use, distribution and reproduction in any medium, provided the original author(s) and source are credited.

\section{References}

Ahn YH, Park J (2007) Efficient visible light detection using individual germanium nanowire field effect transistors. Appl Phys Lett 91:162102-162104

Artoni P, Pecora EF, Irrera A, Priolo F (2011) Kinetics of Si and Ge nanowires growth through electron beam evaporation. Nanoscale Res Lett 6:162-169

Barady RF Jr (1994) Coming to an unsticky end. Nature 368:16-17

Blossey R (2003) Self-cleaning surfaces-virtual realities. Nat Mat 2:301-306

Cassie ABD, Baxter S (1944) Wettability of porous surfaces. Trans Faraday Soc 40:546-551

Castain J (1992) Hand book of X-ray photoelectron spectroscopy edited by (Perkin-Elmer Corp, EenPraire, Minnisota 55344, USA)

Chan CK, Zhang XF, Cui Y (2008) High Capacity Li ion battery anodes using Ge nanowires. Nano Lett 8:307-309

Coffinier Y, Janel S, Addad A, Blossey R, Gengembre L, Payen E, Boukhrroub R (2007) Preparation of superhydrophobic silicon oxide nanowire surfaces. Langmuir 23:1608-1611

Coulson SR, Woodward I, Badyal JPS, Brewer SA, Wills C (2000) Super-repellent composite fluoropolymer surfaces. J Phys Chem B 104:8836-8840

Cui Y, Lieber CM (2001) Functional nanoscale electronic devices assembled using silicon nanowire building blocks. Science 291:851-853

Duan X, Niu C, Sahi V, Chen J, Parce JW, Empedocles S, Goldman JL (2003) High-performance thin-film transistors using semicondcutor nanowires and nanoribbons. Nature 425:274-278

Forrest SR (2004) The path to ubiquitous and low-cost organic electronic appliances on plastic. Nature 428:911-918

Furstner R, Barthlott W, Neinhuis C, Wazel P (2005) Wetting and self-cleaning properties of artificial superhydrophobic surfaces. Langmuir 21:956-961

Irrera A, Pecora EF, Priolo F (2009) Control of growth mechanism and orientation in epitaxial Si nanowires grown by electron beam evaporation. Nanotechnology 20:135601-135607

Jalilian R, Sumanasekera GU, Chanrasekhar H, Sunkara MK (2006) Phonon confinement effect and laser heating effects in germanium nanowires. Phys Rev B 74:155421-155426

Jin G, Tang YS, Liu JL, Wang KL (1999) Growth and study of selforganized Ge quantum wires on $\mathrm{Si}(111)$ substrates. Appl Phys Lett 74:2741-2743

Kim MH, Park YH, Kim I, Park TE, Sung YM, Choi HJ (2010) Self catalytic growth of silicon nanowires on stainless steel. Mater Lett 64:2306-2309
Kumar RR, Yuvaraj D, Rao KN (2010) Growth and charcterization of germanium nanowires by electron beam evaporation. Mater Lett 64:1766-1768

Law M, Goldberger J, Yang P (2004) Semiconductor nanowires and nanotubes. Annu Rev Mater Res 34:83-112

Lee M, Koo J, Chung EA, Jeong DY, Koo YS, Kim S (2009) Silicon nanowire-based tunneling field effect transistors on flexible plsatic substrates. Nanotechnology 20:455201-455206

Liang G, Xiang J, Kharche N, Klimeck G, Lieber CM, Lundstrom M (2007) Performance analysis of a $\mathrm{Ge} / \mathrm{Si}$ core/shell nanowire field-effect transistor. Nano Lett 7:642-646

Mcalpine MC, Ahmad H, Wang D, Heath JR (2007) Highly ordered nanowire arrays on plastic substrates for ultrasensitive flexible chemical sensors. Nat Mat 6:379-384

Molle A, Md. Bhuiyan NK, Tallarida G, Fanciulli M (2006) In situ chemical and structural investigations of the oxidation of $\mathrm{Ge}$ (001) substrates by atomic oxygen. Appl Phys Lett 89:8350483506

Nakajima A, Hashimoto K, Wantanabe T (2001) Recent studies on super-hydrophobic films. Monat Fur Che 132:31-41

Oner D, McCarthy TJ (2000) Ultrahydrophobic surfaces. Effects of topography length scales on wettability. Langmuir 16:77777782

Pecorea EF, Irrera A, Artoni P, Boninelli S, Bongiorno C, Spinella C, Priolo F (2010) Hetroepitaxial growth and facetting of $\mathrm{Ge}$ nanowires on $\mathrm{Si}$ (111) by electron-beam evaporation. Electro Solid State Lett 13:K53-K55

Pei LZ, Zhao HS, Tan W, Yu HY, Chem YW, Wang JF, Fan CG, Chen J, Zhang QF (2010) Low temperature growth of single crystalline germanium nanowires. Mater Res Bull 45:153-158

Qian F, Gradecak S, Yat L, Yen C, Lieber CM (2005) Core/multishell nanowire hetrostructures as multicolour high efficiency light emitting diodes. Nano Lett 5:2287-2291

Shiu JY, Kuo CW, Chen P, Mou CY (2004) Fabrication of tunable superhydrophobic surfaces by nanosphere lithography. Chem Mater 16:561-564

Sivakov V, Heyroth F, Falk F, Andra G, Christiansen S, Cryst J (2007) Silicon nanowire growth by electron beam evaporation: Kinetics and energetic contributions to the growth morphology. Growth 300:288-293

Sutter E, Sutter P (2008) Phase diagram of nanoscale alloy particles used for vapour-liquid-solid growth of semiconductor nanowires. Nano Lett 8:411-414

Tsakalakos L, Balch J, Fronheiser J, Korevaar BA, Sulima O, Rand J (2007) Silicon nanowire solar cells. Appl Phys Lett 91: 233117-233119

Wagner RS, Ellis WC (1964) Vapour-Liquid-Solid mechanism of single crystal growth. Appl Phys Lett 4:89-90

Wang D (2007) Synthesis and properties of germanium nanowires. Pure Appl Chem 79:55-65

Wenzel RN (1936) Resistance of solid surfaces to wetting by water. Indust Eng Chem 28:988-994

Xiang J, Lu W, Hu Y, Wu Y, Yan H, Lieber CM (2006) Ge/Si nanowire hetrostructures as high performance field - effect transistors. Nature 44:489-493

Xu T, Sulerzycki J, Philippe J, Patriarche G, Grandidier B, Stievenard D (2011) Synthesis of long group IV semicondcutor nanowires by molecular beam epitaxy. Nanoscale Res Lett 6:113-119

Yung C, Gau C (2010) Control of superhydrophilicity and superhydrophobicity of a superwetting silicon nanowire surface. J Electrochem Soc 157:K201-K205 